\title{
REVISTA CUESTIONES TEOLÓGICAS (1974-2016) DE LA UNIVERSIDAD PONTIFICIA BOLIVARIANA: 100 números de un proyecto intelectual y teológico de interpretación
}

Cuestiones Teológicas (1974-2016) of Universidad Pontificia Bolivariana: 100 Issues of an Intellectual and Theological Project of Interpretation

Revista Cuestiones Teológicas (1974-2016) da Universidad Pontificia Bolivariana: 100 números de um projeto intelectual e teológico de interpretaçáo

\author{
Camilo Atehortúa Henao* \\ Catalina Castrillón Gallego**
}

* Estudiante del programa de Historia, Universidad Pontificia Bolivariana.

Correo electrónico: camilo.atehortua@upb.edu.co

** Doctora en Historia de la Universidad Nacional, Medellín (2013). Actualmente es la coordinadora del programa de Historia de la Universidad Pontificia Bolivariana.

Correo electrónico: catalina.castrillon@upb.edu.co

Artículo recibido el 15 Noviembre de 2016 y aprobado para su publicación el 10 diciembre de 2016. 


\title{
Resumen
}

El presente artículo presenta una semblanza histórica de la revista Cuestiones Teológicas de la Universidad Pontificia Bolivariana por la conmemoración de sus cien números publicados a lo largo de cuarenta y dos ańos de vigencia académica. Partiendo de la de la revisión y análisis de los 99 ejemplares de la revista, en el marco de los planteamientos de la historia intelectual, se expondrán tres variables que identifican la transformación de esta publicación, desde sus primeros números hasta los más recientes, advirtiendo la riqueza y el carácter de los debates teológicos desplegados en sus diferentes ediciones. Estas variables son: su tránsito de herramienta de divulgación académica e institucional hasta consolidarse en plataforma de debate intelectual a nivel internacional; la conmemoración de efemérides - como el Quinto Centenario del Descubrimiento de América, la promulgación de encíclicas o la celebración de concilios - que avivaron la reflexión en sus autores; y, por último, la relevancia de las perspectivas temáticas de algunos pensadores de la Facultad de Teología. Se pudo constatar que las distintas transformaciones de la revista en el tiempo favorecieron su consolidación académica e intelectual en el debate teológico dentro y fuera de la Universidad Pontificia Bolivariana.

\section{Palabras clave}

Revista Cuestiones Teológicas, Facultad de Teología de la UPB, Historia intelectual, Publicación seriada.

\begin{abstract}
The aim of the article is to present a historical overview of the journal Cuestiones Teológicas of Universidad Pontificia Bolivariana on the publication of the $100^{\text {th }}$ issue after 42 years of existence. Based on a database created with the previous 99 issues and highlighting the academic richness of the theological debates held through all these issues, three variables that characterize the transformation through its years of existence are presented: i) the change from being an institutional means of dissemination to its establishment as an international debate scenario, ii) the commemoration of some important dates (e.g., the Fifth Centenary of the Discovery of America, the publication of some encyclicals,
\end{abstract}


and the celebration of some ecumenical councils) that encouraged the reflection of the authors, and iii) the relevance of the perspectives and topics developed by some of the professors at the Department of Theology. It is argued that the conditions of transformation through time (commemorations were important moments for the authors) and the material conditions (Printing workshops, Publishing houses, among others) that were crucial for the stability of the journal, contributed to the academic and intellectual entrenchment of the theological debate in the University.

\section{Key words}

Cuestiones Teológicas, Department of Theology at UPB, Intellectual History, Serial Publication.

\section{Resumo}

O presente artigo apresenta um esboço histórico da revista Cuestiones Teológicas da Universidad Pontificia Bolivariana, pela comemoração de seus cem números, produzidos ao longo de quarenta e dois anos de vigência acadêmica. Partindo da base de dados construída a partir dos 99 números da revista, expóemse três variáveis que identificam a transformação da publicação, desde seus primeiros números até os mais recentes, advertindo a riqueza e o caráter dos debates teológicos esparsos nas suas diferentes edições. Essas variáveis são: seu processo de câmbio de ferramenta de divulgação acadêmica e institucional até se consolidar como plataforma de debate intelectual a nível internacional; a comemoração de alguns acontecimentos históricos -como o quinto centenário da descoberta de América, a promulgação de encíclicas ou a comemoração de concílios- que avivaram a reflexão em seus autores; e, por último, a relevância das perspectivas temáticas de alguns pensadores da Faculdade. Pode-se constatar que as condiçôes de transformação com respeito ao tempo (as comemoraçóes foram um ponto notável para a reflexão e interpretação dos autores), e as possibilidades materiais (Oficinas de Impressão, Editora, entre outros), que foram fundamentais para a estabilidade da revista Cuestiones Teológicas, favoreceram a consolidação acadêmica e intelectual do debate teológico na Universidad Pontificia Bolivariana. 


\section{Palavras-chave}

Revista Cuestiones Teológicas, Faculdade de Teologia da UPB, História intelectual, Publicação seriada.

\section{INTRODUCCIÓN}

En el año 1974 la naciente Facultad de Teología de la Universidad Pontificia Bolivariana (UPB) presentó, desde la voz y letra de Alberto Ramírez, la revista Cuestiones Teológicas como su órgano oficial (1974, p. 1). Una serie de factores impulsaron la creación de la revista, los cuales remitían a preocupaciones tanto teológicas como sociales:

Existe una conciencia común entre nosotros que revela el deseo de construir una iglesia viva y adulta, conciencia en la que han ejercido un influjo decisivo, como era de esperar, acontecimientos tan importantes como el Concilio Vaticano II y la II Reunión de la Conferencia Episcopal Latinoamericana, esta última realizada precisamente en Medellín (Ramírez, 1974, p.1)

Esta conciencia inscrita en términos posconciliares y de firme relación con el Consejo Episcopal Latinoamericano (CELAM), se articuló a partir de diferentes elementos para concebir la dinámica de funcionamiento de los nacientes años de la revista Cuestiones Teológicas. Uno de los primeros factores para establecer sus bases intelectuales estuvo regido por los estudios en ciencias eclesiásticas que muchos sacerdotes y estudiantes del Seminario Conciliar de Medellín, adelantaron en centros europeos de prestigio académico. Así, se fue robusteciendo no solo el cuerpo del programa académico de la Facultad de Teología, sino, como se mostrará en el desarrollo de este artículo, la revista Cuestiones Teológicas.

Ahora bien, en palabras de Alberto Ramírez, la revista se presentó ante sus lectores (la comunidad de teólogos, estudiantes, profesores de la Facultad, amigos y cristianos interesados) como una posibilidad de contacto con la Facultad de Teología. Es decir, se promovió un marcado reconocimiento de la finalidad y las realizaciones de la Facultad como institución dentro de la Universidad. Los integrantes de la comunidad teológica vinculada a 
la Universidad Pontificia Bolivariana debían cobrar plena conciencia de la misión que les ha sido confiada: "Desde la comunidad cristiana tratamos de convertir la teología en ciencia o conciencia profunda de toda la labor integrada universitaria. Esta conciencia debe actuar como motivación que orienta a todos los hombres hacia la construcción de una sociedad mejor" (Ramírez, 1974b, p. 1).

Desde esta perspectiva, que vincula la especialización académica y la construcción de una conciencia por lo propio, fue que la revista gestó un mecanismo para declarar una postura crítica frente al debate teológico mundial:

Y nosotros tenemos conciencia de que algunos temas básicos, en los cuales trabaja toda la teología universal, pueden ser abordados por nosotros, desde nuestra situación, sin descuidar, de todos modos, el gran patrimonio adquirido y realizado por los otros cristianos, especialmente europeos (Ramírez, 1975, p.1).

Este fue el riesgo intelectual que asumieron quienes integraron la comunidad académica en pos del sostenimiento de la revista Cuestiones Teológicas: enunciar desde el ámbito local, desde Medellín y la UPB, una interpretación de los problemas y debates teológicos que comprendiera el panorama mundial. Esto se evidencia en los criterios tenidos en cuenta para la selección del material académico para ser publicado,

Hemos fijado como criterios fundamentales, entre otros, el de no cumplir simplemente con un deber rutinario, sino el de realizar verdaderamente un esfuerzo creador, que cada vez más vaya haciendo aparecer el carácter original de nuestro trabajo. Queremos hacer conocer solamente artículos que surjan de nuestro medio, no porque desconozcamos el valor de los que son realizados en otras partes, sino porque queremos presentar una contribución verdaderamente nuestra (Ramírez, 1975b, p.1).

Es posible advertir que estos criterios también sirvieron para definir un campo de acción práctico: “[...] realizar un servicio teológico desde nuestra Iglesia local $[\ldots]$ acogemos problemas y situaciones ejemplares, los cuales, iluminados teológicamente, pueden ser entregados a la Iglesia universal" (Ramírez, 1975b, p.1). 
Estas condiciones precisaron, según la intención criteriológica de Cuestiones Teológicas, el carácter de la Facultad de Teología como "instrumento de servicio dentro de la Universidad, dentro de la Iglesia local y en función de toda la Iglesia [...] ella realiza una tarea humana de utilidad real en relación con la construcción de una sociedad mejor" (Ramírez, 1975c, p. 2)

Con este artículo se ha buscado hacer un acercamiento retrospectivo a la revista Cuestiones Teológicas definiéndola como problema de investigación historiográfico dentro de la construcción de un campo de reflexión y producción del saber teológico en Medellín. La historia intelectual permite estudiar temas y problemas asociados a las comunidades de conocimiento y sus respectivos campos reflexivos, a partir de sus productos intelectuales. Basta con señalar las disquisiciones que Michel Foucault y François Dosse aportan al escenario de la historia intelectual.

Para Foucault, la reflexión por el archivo como escenario que "aparte de instituir los acontecimientos archivables en el cruce entre lo nomológico y topológico, resultan también de otro nuevo cruce [...] entre los enunciados que lo conforman y las visibilidades que estos tratan de hacer legibles" (Razón y Sociedad, 2000, p. 176). Es decir, el ejercicio archivístico, previo a esta reflexión escrita, permite evidenciar el cruce entre la voluntad de aquella Facultad de Teología desde 1974, como naciente institución intelectual y la visibilidad alcanzada gracias a la producción de una revista de divulgación de conocimiento teológico y filosófico, como lo fue y es todavía Cuestiones Teologicas.

Al respecto, Dosse (2007), permite apuntalar este panorama teórico cuando expone que,

Sin intención imperial, esta historia intelectual simplemente tiene como ambición el hacer que se expresen al mismo tiempo las obras, sus autores y el contexto que las ha visto nacer, de una manera que rechaza la alternativa empobrecedora entre una lectura interna de las obras y una aproximación externa que priorice únicamente las redes de sociabilidad (p. 14).

En suma, la triada autor, obra y contexto dentro del ámbito de Cuestiones Teológicas, será la posibilidad de cruzar dos líneas de fuerza: una diacrónica 
vertical que vincula la reflexión intelectual de la revista con una tradición teológica de pensamiento, frente a otra sincrónica horizontal que relaciona su contenido con las dinámicas pertenecientes a cada momento histórico.

Por último, es necesario señalar que Cuestiones Teológicas será entendida como campo de acción de un ejercicio moderno de conciencia histórica, es decir, de reflexión crítica de la tradición que recibió en sus manos gracias a su vínculo académico y espiritual con la Universidad Pontificia Bolivariana y la Iglesia Católica.

La conciencia moderna toma -justamente como «conciencia histórica»- una posición reflexiva en la consideración de todo aquello que es entregado por la tradición. La conciencia histórica no oye más bellamente la voz que le viene del pasado, sino que, reflexionando sobre ella, la reemplaza en el contexto donde ha enraizado, para ver en ella el significado y el valor relativo que le conviene. Este comportamiento reflexivo de cara a la tradición se llama interpretación (Gadamer, 1993, p. 43)

A partir de lo anterior se podrá entender el trabajo intelectual de Cuestiones Teológicas desde su inicio, como el producto de una comunidad de interpretación, en pos de una constante búsqueda intelectual en términos teológicos, filosóficos, humanos y cristianos.

En primer lugar, se muestra el recorrido de Cuestiones Teológicas hasta convertirse en una plataforma de debate intelectual a nivel local e internacional y no solamente una herramienta de divulgación académica e institucional, como lo fue en sus primeros ańos. La segunda parte del artículo se ocupará de evidenciar cómo la conmemoración de algunas efemérides avivó la reflexión de los autores en torno a la actualización del debate intelectual de los problemas teológicos. Finalmente, la tercera parte analiza la relevancia temática de algunos pensadores cercanos a la Facultad evidente en las páginas de Cuestiones Teológicas y que permite dar cuenta de algunos rasgos del pensamiento original declarado por Alberto Ramírez en los números iniciales de la revista.

La misión de Cuestiones Teológicas que resaltaba el carácter científico de la teología, a la luz de una conciencia de integración con el mundo universitario, permitirá observar los diferentes cambios que tuvo la revista 
con el paso del tiempo y las condiciones materiales, que favorecieron su estabilidad y consolidación en el escenario académico e intelectual a nivel local e internacional.

\section{DE LA DIVULGACIÓN LOCAL AL DEBATE INTERNACIONAL}

Una de las situaciones más valiosas a la hora de explorar una revista como Cuestiones Teológicas es la evidente transformación que, a través del tiempo, comenzó a presentar frente a la cada vez más lejana referencia del primer número publicado. No solo se trató de cambios relacionados con elementos físicos y gráficos sino también en términos de las intenciones discursivas y del formato mismo. Es decir, se puede verificar una transformación que al mismo tiempo conserva ciertos aspectos esenciales como marca de identidad institucional prefigurada en las primeras entregas de la publicación.

A partir de la revisión de los 99 números publicados de Cuestiones Teológicas, fue posible identificar cuatro periodos que dan cuenta de los mencionados cambios: 1974-1983, 1983-1988, 1988-2001 y finalmente, 2001 hasta el presente. Para ello, se tuvieron en cuenta aspectos como el cuerpo editorial, la organización administrativa, las secciones de la revista y las imprentas o editoriales con las que ha tenido relación.

Para el primer periodo (1974 y 1983) es posible advertir continuidad en cuanto a la concepción de Cuestiones Teológicas como una publicación vinculada intelectual y teológicamente con el CELAM y el Concilio Vaticano II, que además buscaba ser un escenario de contacto al interior de la Facultad de Teología de la Universidad Pontificia Bolivariana. A esto se sumaba la intención de convertir la teología en ciencia, la integración universitaria desde la reflexión teológica, la conciencia histórica ante el debate teológico mundial y la presentación de una voz propia y original en el ámbito teológico.

El primer número de Cuestiones Teológicas estaba divido en tres secciones: Entrega, Tema Central y Estudios Teológicos. Sobre esta triada se fueron añadiendo otras secciones. En unos pocos números posteriores comenzó a aparecer una Sección Bibliográfica en la que se reseñaban y comentaban 
libros como los del argentino Enrique Dussel y el húngaro Eugenio Lakatos que, en su calidad de sacerdote de la Congregación del Verbo Divino, acompañó al Humberto Jiménez en aquella tarea de consolidar y poner en marcha el movimiento bíblico que dio paso al Instituto de Estudios Bíblicos de la Universidad de Antioquia.

Durante este periodo, Cuestiones Teológicas también dio espacio a un evento que por entonces tomaba el nombre de Seminario Permanente y que relacionaba los avances académicos en torno a las reflexiones realizadas dentro de la Facultad sobre temas como la ética y la ciencia. Así mismo, era frecuente la publicación de números dedicados por completo a eventos académicos como el III Congreso de Teología de 1977 celebrado en Bucaramanga.

Además, en aras de su constitución como escenario científico del conocimiento teológico desde una mirada propia, Cuestiones Teológicas emprendió la divulgación de las tesis doctorales elaboradas por miembros de la Facultad en otras tradiciones académicas. Tal fue el caso de Fernando José Bernal y su trabajo "De la fé en el mundo a la fé en Dios", reflexión a partir de Teilhard de Chardin; o el de Gonzalo Soto Posada sobre "La función de las etimologías en San Isidoro de Sevilla”.

Ahora bien, los temas de carácter académico-administrativo sobre planeación educativa dentro del programa de Teología, los informes de decanatura y los planes de desarrollo, también tuvieron espacio en las páginas de los primeros números. Ampliando esa noción de "contacto" que parecía restringida de alguna manera al ambiente netamente académico, la revista también funcionó como órgano informativo de actualidad sobre los acontecimientos de interés que comprometían a la Facultad misma.

En cuanto a los cargos administrativos, este periodo estuvo marcado por una sencilla distribución de las responsabilidades editoriales de la revista. El cargo de Jefe de Redacción, ocupado por Alberto Ramírez hasta 1988, era la voz de mando. Le acompańó con el paso de las entregas un Cuerpo de Redacción del que hacían parte los coordinadores de los distintos departamentos de la Facultad de Teología. Hacia 1979, este cuerpo tendría nombres propios como Gonzalo Restrepo, Néstor Giraldo, Andrés Rosero y el mismo Alberto Ramírez. 
Durante este primer periodo se hizo evidente la dificultad relacionada con la periodicidad de la revista. Aunque su intención declarada era la de aparecer tres veces al año, no era extraño encontrarse con números extraordinarios, que se encargaban de compendiar ediciones anuales que por diversos motivos, no salieron con puntualidad a manos de los lectores. Por otro lado, durante este periodo, Cuestiones Teológicas pasó por los siguientes talleres de impresión: Granamérica (1974), Servigráficas (1975-1977, 1979), Talleres tipográficos de la UPB (1978,1980), Quimara Ltda (1981-1985). En comparación con los periodos posteriores, esta situación da muestras de una inestabilidad en la periodicidad hasta la consolidación de la Editorial UPB como encargada de su publicación alrededor de 1987.

Igualmente, en este periodo confluyen momentos relevantes para la revista, la Facultad y la Universidad. En 1978 Cuestiones Teológicas obtiene su número de identificación internacional (ISSN 0120-131x); luego, en 1981, la Facultad de Teología define su situación ante las autoridades educativas del país al obtener la licencia por acuerdo No. 150 del 19 de agosto de 1980 emitida por ICFES, que favorecía su consolidación como programa académico. Otro hecho importante fue la aprobación canónica de la Facultad de Teología, bajo decreto de 25 de mayo de 1980 de la Sagrada Congregación para la Educación Católica, en el número 19 de 1980 se publicó el documento oficial.

A partir de 1983, se integra a la revista la Facultad Eclesiástica de Filosofía, dando inicio al segundo periodo que se extiende hasta 1888. En términos formales, se divide en dos partes, cada una dedicada a una Facultad. Continuó la publicación de números dedicados a eventos académicos, tesis doctorales de personas vinculadas a ambas facultades y de la Sección Bibliográfica, aunque esta última, con bastante irregularidad.

Este breve periodo de transición y acoplamiento de la presencia de la Facultad de Filosofía como parte de la revista Cuestiones Teológicas, mostró el camino hacia la apropiación de los procesos técnicos y editoriales desde la Universidad Pontificia Bolivariana, iniciados en 1985 y retomados en 1987 después de un breve paso por los talleres de Quimara Ltda. 
Durante este periodo tuvo lugar la conmemoración de los 50 años de la Universidad Pontificia Bolivariana; el número 35 de 1986 dio cuenta de esto y de la visita del Papa Juan Pablo II a Medellín. Fue una posibilidad de construir nuevamente el relato de la historia de la Facultad de Teología y su relación con el quehacer teológico y filosófico.

En el año de 1988, Alberto Ramírez terminó su ciclo como director de Cuestiones Teológicas y se da inicio al tercer periodo que se extiende hasta 2001. En adelante es posible advertir la consolidación del carácter científico e internacional de la revista, la expansión de su alcance inicial y su apertura hacia nuevos horizontes intelectuales.

Las secciones tomaron un cariz pragmático desde la llegada a la dirección de la revista de Néstor Giraldo. Su estructura se simplificó. Ahora solo contaba con una breve presentación, que en algunos casos se nombró como "editorial" y en otros simplemente se omitió, y con un tema central que acogía la totalidad de los artículos publicados en cada número. A partir de este momento no vuelven a aparecer en sus páginas ninguna tesis doctoral o documentos académico-administrativos. En la década de 1990 se normalizó la periodicidad de publicación a dos números por año, y desde 1997, todos los artículos presentados por la revista, comenzaron a contar con un resumen acompañado de palabras clave.

La Sección Bibliográfica tuvo una presencia intermitente hasta perderse por completo En la década del 2000 reapareció con un carácter distinto. Además, desde 1994 se introdujeron secciones nuevas, como por ejemplo, Noticias de las Facultades y Eventos. De igual forma, este año el nombre de la revista pasó de ser el de Cuestiones Teológicas a Cuestiones Teológicas y Filosóficas para así refrendar los lazos de hermandad anunciados con antelación.

Desde 1998 aparecen dos nuevas secciones. Una, llamada Notas y Comentarios y otra, sobre lenguas clásicas conocida como Sección Di Lingua que promovía el conocimiento del latín y del griego, este último a cargo de Jorge Iván Aguirre. En 1999 la sección de Estudios formalizó la presencia de los resultados de las investigaciones adelantadas por miembros de la Escuela haciendo eco a la presencia de las tesis doctorales publicadas en anteriores periodos de la revista. Podría decirse que de esta manera se atendía a la necesidad de continuar plantando la voz propia, el riesgo interpretativo. 
En cuanto a lo administrativo, fueron varios los directores durante este periodo. A Néstor Giraldo lo reemplazó Gonzalo Restrepo en el año de 1994. En 1997, Álvaro Cadavid asumió las riendas hasta el año 2002. En 1994 el Cuerpo de Redacción se consolidó con nombres como los de Julio Jaramillo o Argiro Restrepo y, asimismo, se vinculó un nuevo puesto dentro de este órgano administrativo de la revista. Se trató del cargo de Coordinador Operativo. Así, inició la preparación para dar el salto internacional que se advirtió cuando en el año de 1999 se anexó a este cuerpo administrativo, el Consejo Internacional con representantes de universidades de Alemania, Brasil y Chile.

En 1996 apareció uno de los primeros trabajos realizados por un autor extranjero, el profesor chileno Ciro Schmidt Andrade. Aunque en números anteriores se publicaron artículos traducidos, este hecho habló del cambio acaecido frente a los criterios de funcionamiento de Cuestiones Teológicas, que exigían en la década de 1970 la presencia exclusiva de trabajos realizados por personas pertenecientes a la Facultad, a la Universidad.

Durante el 2001 la revista obtiene de parte de COLCIENCIAS el reconocimiento como publicación de índole científica, dando inicio al último periodo, que se extiende hasta la actualidad. En adelante, Cuestiones Teológicas definió su campo de estudio dentro de la teología y las ciencias humanas y sociales, y las secciones se mantuvieron prácticamente invariables. Quizá lo más llamativo de este periodo se encuentre en su organización administrativa.

A partir de 2001, su organización está compuesta por los cargos de: Director, Editor, Comité de Redacción, Comité Editorial, Consultores Internacionales, Traducciones y Coordinador operativo y administrativo. Años más tarde, aparece el listado una lista con los arbitros para cerrar esta reestructuración dentro de una nueva faceta como revista científica presente en diferentes bases de datos locales e internacionales.

Fue un cambio considerable con respecto a las condiciones operativas expuestas en los periodos anteriores; una transformación que no permite superponer con transparencia un número de la primera década de publicación de la revista con uno de este periodo científico. 
En términos editoriales, fueron pocas cosas las que cambiaron. Tan sólo se marcó la vinculación de varios talleres tipográficos como complemento al trabajo realizado en la universidad por la Editorial UPB. En lo administrativo, Álvaro Cadavid fue sucedido en el cargo de director por Hernán Darío Cardona entre 2003 y 2009 quien a su vez, fue reemplazado por Jairo Alberto Henao y Diego Alonso Marulanda Vélez.

En el año 2004 la revista cambió de nuevo su nombre, por Cuestiones Teológicas, situación que vinculó su campo de aplicación directamente al mundo teológico. Este periodo es el más complejo de revisar en términos históricos por su inevitable cercanía temporal. Quizá visto desde el contraste con las condiciones y valores encontrados en las primeras dos décadas de la revista, sirva como elemento definitorio y aclaratorio de las intenciones prefiguradas durante los momentos iniciales de su publicación.

\section{CONCIENCIA HISTÓRICA Y CONMEMORACIONES}

Es momento de revisar cómo fue tomada la tradición teológica, como voz que vino del pasado, para ser interpretada desde las posibilidades de enunciación que se encontraron alrededor de la revista Cuestiones Teológicas. A partir de la exposición de los hitos históricos asumidos de manera reflexiva por la por la revista y sus autores, se podrá evidenciar aquella premisa ya compartida, que sentenciaba la necesidad de escribir desde lo local las apreciaciones sobre los grandes temas de escala global. Además, la presencia y participación de la revista en eventos académicos marcó la vía de transmisión de ese pensamiento original y especializado que se fue amalgamando, por lo que evocar algunos detalles al respecto favorecerá la discusión sobre el carácter de la revista. Por último, asumiendo cada elemento señalado como participe de la construcción de una consciencia histórica propia, no está de más presentar algunos elementos narrativos sobre el pasado de la Facultad, la Universidad, instituciones amigas y personas que tras su muerte fueron recordadas por su contribución a la vida universitaria y pastoral.

En cuanto a los momentos históricos que fueron dignos de ser interpretados desde las páginas de la revista Cuestiones Teológicas, de la mano 
de autores propios, la lista es extensa. Se pueden encontrar los rastros del seguimiento que dentro de la revista se fue consolidando a partir de dos tipos de reflexiones: una que retomaba documentos de la tradición católica y otra que asumía la presentación de los documentos recientes dentro de la comunidad católica.

Por ejemplo, en el año 1981, con los 90 años de la encíclica Rerum Novarum de León XIII, la revista presentó reflexiones a propósito de la Doctrina Social de la Iglesia, su proceso histórico de gestación y su relación con el contexto local. 10 años más tarde, en 1991 cuando alcanzó los 100 años, el número 49 de 1991 expuso el mismo tema.

La misma situación se presentó alrededor de los 200 años de los Derechos del Hombre y del Ciudadano en 1990; con el centenario de la encíclica Procedentissimus Deus de León XIII en 1993; los 30 años de la Gadium et Spes, la constitución pastoral del Concilio Vaticano II, en 1995; los 40 años del Concilio Vaticano II en 2003. En cada caso, se puede evidenciar la voluntad interpretativa que enuncia la revista Cuestiones Teológicas como una muestra de su vinculación reflexiva con los problemas globales que atañen a la Iglesia Católica. La fecha no fue más que el pretexto para plasmar una mirada original, es decir, para construir un escenario de divulgación de las ideas y esfuerzos que las personas vinculadas a la Facultad de Teología emprendían desde la especialización de sus saberes.

También es posible advertir un proceso similar en términos de documentos eclesiásticos actuales, es decir, que fueron asimilados inmediatamente después de su publicación oficial. Resaltan, por ejemplo, la encíclica Laboren Exercens de 1982, la Centesimus Annus de 1991 o la Veritas Splendor de 1993. Fue la posibilidad de actualización pastoral e intelectual del curso de la reflexión teológica.

Pero no solo fueron las fechas y documentos teológicos los que promovieron este impulso interpretativo. En 1992, con motivo de los 500 años del llamado Descubrimiento de América, la revista enseñó nuevamente un número que dio cuenta de cuáles eran las condiciones interpretativas con las que quería enfrentar la historia y el mundo. El número 51 de la revista, fue un esfuerzo por enlazar la historia con la teología. Todos sus artículos buscaron vincular las condiciones superpuestas entre el mundo indígena y 
el mundo cristiano. Un ejercicio que relacionó la problematización de la evangelización y del encuentro misional con las culturas prehispánicas, en suma, de la relación entre cultura y evangelización. Lo llamativo de este número, al margen de las connotaciones obvias vinculadas a la distancia temporal con 1492, fue la apuesta por asumir bajo la mirada teológica, una interpretación del mundo y su historia. Como si aquel texto inaugural de Alberto Ramírez sobre la historia vista a la luz de la Teología, en este número alcanzara su expresión práctica y teórica.

En cuanto a los eventos académicos, la lista también es extensa. Comenzando por el CELAM en Puebla en el año 1979, la revista inició una expansión del contacto académico con los eventos más importantes a nivel local, nacional e internacional. Los sendos Congresos Nacionales de Teología pueden rastrearse sin ningún problema dentro de las páginas de la revista. Los Seminarios Bíblicos de la Arquidiócesis de Medellín, los Seminarios Permanentes dentro de la Universidad Pontificia Bolivariana, las Semanas Permanentes del Episcopado Colombiano, los Coloquios Universitarios, entre otros. En el año de 1995 se dio razón de la participación en el Congreso Teológico realizado en Santiago de Chile como un encuentro de Facultades de Teología Latinoamericanas que consignó un momento de crecimiento académico a nivel internacional en tanto centro teológico local y que, más tarde, se vería reflejada con la internacionalización de la revista y su consolidación como escenario de una mirada científica de la teología.

La revista, además, se permitió plantear su propia narración de la historia de la Facultad de Teología y de la Universidad Pontificia Bolivariana en los años: 1974, su inauguración; 1980, aprobación canónica del programa; y 1986, con los 50 ańos de la Universidad. Estas conmemoraciones institucionales fueron un motivo imposible de ignorar para representar nuevamente el alcance de esa conciencia histórica prescrita como pilar indeleble de Cuestiones Teologicas y la Facultad. Ahora bien, también dio espacio para la revisión de la memoria histórica del centenario Lasallista o la Universidad Javeriana, lo cual la vinculaba con su contexto de redes de sociabilidad académica y pastoral.

Por último, con motivo del robustecimiento de una identidad propia, también se desplegó un escenario de reposo y reconocimiento para quienes partieron de este mundo dejando un legado digno de ser recordado: David 
Arango Berrio (1935-1981), René Uribe Ferrer (1918-1984), David Kapkin (1940-2012), Humberto Jiménez (1929-2013) y Alberto Ramírez (19402015), fueron algunos de los hombres que la revista acompañó con un homenaje escrito, como una firma ante el tiempo, que como en una puerta perdurable evitará ser alcanzada por el olvido. Parte de sus obras descansan en las páginas de la revista esperando ser estudiadas y releídas, criticadas e interpretadas.

\section{AUTORES Y TEXTOS}

Recordando aquella distinción que planteaba la revista en sus inicios sobre la búsqueda de un tono científico de la teología y de un escenario práctico para la aplicación del conocimiento teológico en la Iglesia local, se pueden relacionar algunas de las perspectivas temáticas que es posible encontrar. Esto como acercamiento a la producción intelectual de la revista desde sus autores, y que servirá de complemento a la presentación de las conmemoraciones históricas que marcaron una clara relación con las reflexiones que se fueron presentando.

Fueron muchos los autores que entregaron sus trabajos al público lector de Cuestiones Teológicas para así conformar, con el paso de los años, una cifra en torno a los mil textos publicados. Sin embargo, para este recuento temático, sólo serán considerados algunos autores que acompañaron con sus textos, en alguna medida, los cuarenta y dos años de vigencia y transformación de la revista Cuestiones Teológicas.

Los dos primeros hombres en reseñar son los Padres Humberto Jiménez y David Kapkin Ruiz. Maestro y discípulo respectivamente, fueron la muestra de la evidente especialización en el campo teológico que fue el cuerpo académico de la Facultad de Teología y, vale mencionarlo, el Instituto de Estudios Bíblicos de la Universidad de Antioquia.

Si bien un texto podría no ser más que la cara transparente que tiene el lector cuando se enfrenta ante un autor, bajo él subyacen no solo condiciones propias de los estudios del discurso, sino, la vida misma de quien escribe. Sus gustos y disgustos, esfuerzos y búsquedas; en suma, la experiencia que 
lleva consigo a cada lugar. De allí la importancia de vincular el tema de estudio con la experiencia cotidiana.

El padre Humberto Jiménez desde la primera década de vida de la revista Cuestiones Teológicas presentó su trabajo teológico de manera constante. Desde los estudios sobre la Sagrada Escritura, fundamentados en su experiencia académica en Roma, comenzó a gestar un territorio de reflexión a partir de comentarios bíblicos. Se recuerda su esfuerzo por establecer las bases de análisis en torno a los profetas, las bases cristológicas, las reflexiones alrededor de la hermenéutica bíblica y la pragmalingüística. Además de la apropiación de autores como Teilhard de Chardin que enriquecieron sin duda a los lectores de la revista Cuestiones Teológicas.

Humberto Jiménez, como recordará Alberto Ramírez, fue un puntal para la introducción de la moderna investigación bíblica en el ámbito local. Además, no solo se encargó de perfilar con constancia los pormenores de sus pensamientos dentro de los marcos del texto escrito, sino que su vocación pastoral lo llevó a transformar el formato de divulgación de su reflexión alcanzando así la radiodifusión en la emisora cultural de la Universidad de Antioquia con el programa "Encuentros con la Biblia" y una activa posición frente el desarrollo del ecumenismo en Colombia (Ramírez, 2014, p. 4).

David Kapkin, quien estuvo presente en el Instituto de Estudios Bíblicos de la Universidad de Antioquia en calidad de estudiante, acompañó también de manera constante el crecimiento de la revista Cuestiones Teológicas. Desde la primera década de sus publicaciones se pueden rastrear las luces de su trabajo, el perfil de su pensamiento. Vinculado abiertamente con una intensa apropiación de la ciencia, el psicoanálisis y la literatura, que mereció el reconocimiento del por entonces profesor Ratzinger, posterior Papa Benedicto XVI, encaminó sus esfuerzos a realizar una amplia gama de comentarios bíblicos como sus "Cuestiones en torno a la pasión, muerte y resurrección de Jesús" (Kapkin, 1987) o "El ocaso del cristianismo y la eterna Aurora de la religión" (Kapkin, 1993) que fue una lectura de la Biblia desde las posibilidades psicoanalíticas que un autor como Eugen Drewermann le entregó. Complementó su producción intelectual con varios libros como 1 Corintios: un iglesia inquieta, Marcos: Historia humana del hijo de Dios (Kapkin, 1997), entre otros. En Kapkin fue marcada la apropiación de los elementos de la lingüística, el conocimiento de lenguas clásicas y, además, 
una activa presencia en la radio, al igual que Humberto Jiménez, esta vez desde la emisora La Voz de Jesucristo de la Escuela Bíblica.

Además de los esfuerzos presentados por Humberto Jiménez y David Kapkin, es valioso hacer hincapié en la producción intelectual de Alberto Ramírez, que puede ser estudiada a la luz de esa noción sobre lo original del pensamiento propio, bolivariano, impulsado por la revista. Ahora bien, sobre Alberto Ramírez, entendiendo sus casi cien textos publicados en la revista Cuestiones Teológicas sumado a sus trabajos con otro tipo de formato, sólo se presentará una faceta sencilla pero vital y, sin duda, llena de posibilidades de reflexión sobre la identidad y la búsqueda de la voz propia cuando se trata de un colectivo intelectual, espiritual y humano como lo fue y lo es la Facultad de Teología y su órgano oficial. Esa faceta fue la reflexión histórica con la que acompañó, como guardián del tiempo, el discurrir de los años la Facultad de Teología.

Los primeros artículos que se pueden encontrar en el número inaugural de la revista Cuestiones Teológicas escritos por Alberto Ramírez son totalmente dicientes al respecto: "Origen y significación de una nueva Facultad de Teología en la Universidad Pontificia Bolivariana” y "El problema de la historia a la luz de la Teología" (Ramírez, 1974). Fue desde su reflexión que se comenzó a gestar una base firme para la construcción de una conciencia histórica resistente ante el paso del tiempo, evidente con cada página publicada al respecto. No solo se permitía relacionar los pormenores sobre la historia de la Facultad de Teología o la revista misma, sino que acercaba la conmemoración de acontecimientos como en su momento lo fueron, por ejemplo, los diez años del CELAM en Medellín y el CELAM en Puebla, en 1978. También fue notable el hilo con el que comenzó a enlazar la tradición teológica universal con la reflexión local sobre los documentos más notables elaborados en la Iglesia Católica. Por su pluma fueron patentes las reflexiones históricas en torno al contexto de gestación de encíclicas, concilios, entre otros. Verbigracia, "Medellín y el origen reciente de la vocación profética de nuestra Iglesia en América Latina” (Ramírez, 1998) y "A los 40 años del Concilio Vaticano II" (Ramírez, 2003), fueron algunos de sus artículos en torno a tal condición historiográfica.

Lo que sobró en la reflexión histórica de Alberto Ramírez fue la necesidad incontestable de actualizar constantemente el debate teológico 
desde la perspectiva de la localidad alojada en la Facultad de Teología. Como si desde el primer número de la revista ya tuviera conciencia de su lugar de vigía del tiempo, este hombre, se encargó de narrar "La Tradición histórica de nuestra Facultad" (Ramírez, 2014) y los "Datos históricos sobre el origen de la Facultad de Teología" (Ramírez, 1986).

En ningún momento la intención expuesta pretende reducir o simplificar el extenso conocimiento reflejado en la multiplicidad de la obra de Alberto Ramírez, entre continuidades y discontinuidades pero, para el momento que nos atañe alrededor de los cien números de la revista Cuestiones Teológicas, es inevitable ver cómo la figura de este hombre exige una prolongación narrativa; una renovación del esfuerzo por describir permanentemente cómo la historia y los seres humanos se transforman.

Hasta aquí, se han señalado temas variables cercanos a los comentarios bíblicos, la apropiación de autores notables como Chardin y Drewermann, el vínculo intelectual con la práctica pastoral y la historia. Vale entonces completar este breve horizonte reflexivo, que desafortunadamente no puede incluir a muchos más autores, con el rostro femenino de la teología y el rostro filosófico de la revista. Se hace referencia a Lucia Victoria Hernández, Marta Inés Restrepo y a Gonzalo Soto Posada.

Lucia Hernández fue una de las más notables estudiantes que haya tenido el Padre Humberto Jiménez y una fiel compañera del proceso del crecimiento de la revista al igual que Marta Inés Restrepo. Estas dos mujeres enfocaron sus esfuerzos teológicos en asumir el problema de la religiosidad, ya fuera desde la figura del niño y su formación académica, como lo presentó Marta Inés Restrepo, o desde la complejidad de lo "popular" como lo muestran los esfuerzos de Lucia Hernández por estudiar los símbolos en las fiestas navideńas. Sus reflexiones no se agotaron en este tema preciso dentro de la revista Cuestiones Teológicas, pues también se vieron reflexiones sobre la mujer en el Antiguo Testamento o sobre la mujer después del Concilio Vaticano II; sin embargo, fueron entregas recurrentes que se pueden advertir con el paso de los números y el tiempo.

Con Gonzalo Soto es visible una de las condiciones de configuración de una voz propia desde esa declaración por lo nuestro expuesta en la revista. La presencia de su tesis doctoral "Las etimologías de San Isidoro de Sevilla" 
(Soto, 1980) presentada en la Pontificia Universidad Gregoriana de Roma, marcó sin duda la participación filosófica dentro de la revista y la recepción del proceso de especialización académica adelantado por los representantes de la Facultad de Teología en el ámbito global.

La creación de un espacio propio para la reflexión sobre las condiciones medievales del pensamiento, que no permiten discernir con solvencia la Teología de la Filosofía, fortaleció el debate dentro de la naciente Facultad Eclesiástica de Filosofía de los años 1980 y creó referentes temáticos como la Estética Medieval, Filosofía y Trinidad, Santo Tomás e Isidoro de Sevilla para robustecer el debate dentro de la revista Cuestiones Teológicas y el mundo académico circundante.

También, es importante recordar el trabajo de Monseñor Darío Múnera Vélez, Augusto Uribe y Néstor Giraldo que registran una amplia producción dentro de la revista desde los primeros años de la revista Cuestiones Teológicas hasta la primera década del siglo XXI. Es un trabajo digno de un estudio juicioso y pormenorizado sobre cómo se apropió el pensamiento y obra de autores como Marie-Joseph Lagrange, y Hans Kung; sobre la propuesta interpretativa de las ideologías o el sentido del humanismo y la enseñanza social de la Iglesia. Son muchos más los autores y autoras que nutren las páginas de la revista Cuestiones Teológicas durante estos 42 años de vigencia intelectual y pastoral por lo que esta no es más que una invitación a reivindicar la originalidad del pensamiento que descansa en los anales de la Facultad de Teología de la Universidad Pontificia Bolivariana.

\section{CONCLUSIÓN}

Cuando Alberto Ramírez señaló que la revista Cuestiones Teológicas se erigiría en órgano oficial de la Facultad de Teología, enmarcó las condiciones de existencia de 42 años de camino. La relación que ella tuvo con la Facultad de Teología es afortunada e inevitable. Se convirtió en el formato de transmisión de una voz propia, nueva en el medio local, que fue tomando fuerza hasta alcanzar escenarios que quizá no se veían con claridad durante los primeros años de publicación. Esa transformación de la noción de contacto, 
que precisaba la relación con la Facultad y que alcanzó la transmisión del conocimiento propio, fue una de las consecuencias del paso del tiempo por sus páginas. Cambió el carácter, la periodicidad, el formato, los criterios de publicación, el nombre, pero no cambió, en lo esencial, el vínculo con la Facultad, su intención de integración universitaria ni esa necesidad de rigor científico desde la reflexión teológica.

Además, la constante recuperación del pasado propio y global, la llevó a preguntarse sobre los problemas que incumben a la humanidad misma, lo cual le permitió sentar las bases de una reflexión independiente desde el mundo latinoamericano favorecido desde el siglo XX por el Concilio Vaticano II y el CELAM. Ese vínculo con el pasado, esa necesidad histórica de narrarse dentro de la revista, fue una condición que vista de manera panorámica plantea una ruta de identidad y reconocimiento del gran esfuerzo académico, humano y pastoral de las personas que hicieron parte y aun hacen parte de la facultad de Teología y de la Universidad Pontificia Bolivariana.

La revista, igualmente, como se puede advertir en el cuadro anexo, fue un lugar de participación académica e institucional de muchas de las personas que con los años hicieron parte del mundo directivo de la universidad. Los casos de Gonzalo Restrepo Restrepo y Darío Múnera Vélez dan cuenta de que la Facultad, representada por la revista, fue una cantera de líderes prestos a tomar las riendas administrativas del mundo académico. No queda más que hacer una intensa invitación a la relectura y apropiación, valiente y rigurosa, del pensamiento que se conserva en las páginas de una revista. Una invitación a estudiar las condiciones históricas del debate teológico del que participó y que merecen ser profundizadas. Este artículo es un aporte a la celebración de los cien números de la revista Cuestiones Teológicas marcados por la vigencia académica en el mundo teológico desde su apuesta por un pensamiento original y propio.

\section{LISTA DE REFERENCIAS}

Revista Cuestiones Teológicas N. 1-99 
Dosse, F. (2007). La marcha de las ideas: historia de los intelectuales, historia intelectual. Valencia: Publicacions de la Universitat de Valencia.

Foucault, M. (1970). Arqueología del saber. México: Siglo XXI.

Gadamer, H. G. (1991). El problema de la conciencia histórica . Madrid: Editorial Tecnos.

Velemazán, P. P. (2000). Los Archivos de Michel Faocault . In J. M. Pablo López Álvarez, La impaciencia de la libertad. Michel Faoucault y lo politico (pp. 161-199). Madrid: Biblioteca Nueva. 


\section{ANEXO}

Nexo Cuestiones Teológicas con cargos académico- administrativos en la Universidad Pontificia Bolivariana

\begin{tabular}{|c|c|c|}
\hline Nombre & $\begin{array}{l}\text { Participación en la } \\
\text { revista }\end{array}$ & $\begin{array}{l}\text { Cargo } \\
\text { academico-administrativo }\end{array}$ \\
\hline $\begin{array}{l}\text { Darío Múnera } \\
\text { Vélez }\end{array}$ & Autor & $\begin{array}{l}\text { Decano Facultad de Teología: } \\
\text { 1978-1982 } \\
\text { Rector UPB: 1989-1997 }\end{array}$ \\
\hline $\begin{array}{l}\text { Gonzalo Soto } \\
\text { Posada }\end{array}$ & $\begin{array}{l}\text { Autor } \\
\text { Dirección de Revista: } \\
1983\end{array}$ & $\begin{array}{l}\text { Decano Facultad Ecca. de } \\
\text { Filosofía: 1987-1997 } \\
\text { Cuerpo de redacción: 1994- }\end{array}$ \\
\hline $\begin{array}{l}\text { Gonzalo Restrepo } \\
\text { Restrepo }\end{array}$ & $\begin{array}{l}\text { Cuerpo de redacción } \\
1979-1996 \\
\text { Director de la revista: } \\
1994-1997\end{array}$ & $\begin{array}{l}\text { Jefe de Facultad: } 1979-1996 \\
\text { Decano de la Facultad Ecca de } \\
\text { Filosofía: } 1983-1987 \\
\text { Rector UPB: } 1997-2004\end{array}$ \\
\hline Guillermo Zuleta & Autor & $\begin{array}{l}\text { Decano de Teología: } 1999 \\
\text { Decano Esc. TFH: 2013-2016 }\end{array}$ \\
\hline Emilio Betancur & Autor & $\begin{array}{l}\text { Decano Facultad Teología : 1982- } \\
1987\end{array}$ \\
\hline $\begin{array}{l}\text { José Fernando } \\
\text { Bernal }\end{array}$ & Autor & $\begin{array}{l}\text { Decano Facultad Teología: 1987- } \\
1996\end{array}$ \\
\hline $\begin{array}{l}\text { Carlos Arboleda } \\
\text { Mora }\end{array}$ & Autor & $\begin{array}{l}\text { Decano Encargado: } 1998 \\
\text { Comité de redacción: } 2001\end{array}$ \\
\hline $\begin{array}{l}\text { Diego Alonso } \\
\text { Marulanda }\end{array}$ & $\begin{array}{l}\text { Autor } \\
\text { Director de la } \\
\text { Revista: } 2010\end{array}$ & $\begin{array}{l}\text { Decano Esc. TFH: 2007-2013 } \\
\text { Vicerrector Pastoral: } 2013 \text { (en } \\
\text { ejercicio) }\end{array}$ \\
\hline $\begin{array}{l}\text { Iván Darío Toro } \\
\text { Jaramillo }\end{array}$ & $\begin{array}{l}\text { Editor de la revista: } \\
2015 \text { hasta la } \\
\text { actualidad }\end{array}$ & $\begin{array}{l}\text { Director Facultad de Teología: } \\
2016\end{array}$ \\
\hline
\end{tabular}

Fuente: Revista Cuestiones Teológicas No. 1-99 